\title{
Severe Focal Stenosis of the Abdominal Aorta with High Risk of Occlusion
}

\author{
Emil-Marian Arbănași1, Adrian Vasile-Mureșan ${ }^{1,2}$, Eliza Russu1,2, Eliza-Mihaela Arbănași3 \\ 1 Clinic of Vascular Surgery, Emergency County Hospital, Târgu Mureș, Romania \\ 2 First Department of Surgery, "George Emil Palade” University of Medicine, Pharmacy, Science and Technology, Târgu Mureș, Romania \\ 3 Faculty of Pharmacy, "George Emil Palade” University of Medicine, Pharmacy, Science and Technology, Târgu Mureș, Romania
}

\section{CORRESPONDENCE}

\section{Eliza Russu}

Str. Gheorghe Marinescu nr. 50

540136 Târgu Mureș, Romania

Tel: +40 744766597

E-mail: eliza_ion@yahoo.com

\section{ARTICLE HISTORY}

Received: September 29, 2021

Accepted: December 29, 2021
Emil-Marian Arbănași • Str. Gheorghe Marinescu nr. 50, 540136 Târgu Mureș, Romania. Tel: +40 758530 111, E-mail: emilarbanasi1@gmail.com

Adrian Vasile Mureșan • Str. Gheorghe Marinescu n 50, 540136 Târgu Mureș, Romania. Tel: +40 744894 319, E-mail: muresanadi@yahoo.com

Eliza-Mihaela Arbănași • Str. Gheorghe Marinescu nr. 50, 540136 Târgu Mureș, Romania. Tel: +40 740974 049, E-mail: arbanasi.eliza@gmail.com
We present the case of a 58-year-old male patient, chronic smoker, with a history of hypertension and diabetes, referred to the Vascular Surgery Unit for claudication below $100 \mathrm{~m}$ (Rutherford III class) and paresthesia in the lower limbs. Physical examination revealed diminished bilateral femoral pulses.

A computed tomography angiography scan revealed severe isolated stenosis (over 70\%) of the abdominal aorta (arrows in Panel A, B, and C), below the emergence of the inferior mesenteric artery, due to a soft plaque, with high thrombogenic risk.

A successful endovascular intervention was performed, via a transfemoral approach, with placement of a 14-mm stent graft in the abdominal aorta, achieving flow restoration, without any intraprocedural events. The postoperative evolution did not record any complications, and the patient was discharged on the third day, symptom-free.

The bifurcation of the aorta is a common vascular site for atherosclerotic lesions, with severe stenosis or occlusion of the abdominal aorta. Standard therapy for these lesions is surgical repair, with endarterectomy for focal aortic disease, or bypass graft placement in case of widespread aortoiliac atherosclerotic disease. The long-term outcomes of surgery are well established in the literature, with graft patency rates of $90 \%$ at five years and $75 \%$ at 10 years, with an early significant complication risk of $5-10 \% .{ }^{1}$

However, because the frequency of focal distal aortic stenosis without involvement of the aortic bifurcation is relatively rare, these lesions are typically documented in the literature in conjunction with stenosis affecting the aortoiliac junction. ${ }^{2}$ Percutaneous transluminal angioplasty (PTA) is used to treat abdominal localized stenosis, ${ }^{3}$ with positive short- and long-term outcomes. PTA is a less invasive alternative to surgery, with less side effects. ${ }^{4}$

\section{CONFLICT OF INTEREST}

Nothing to declare. 

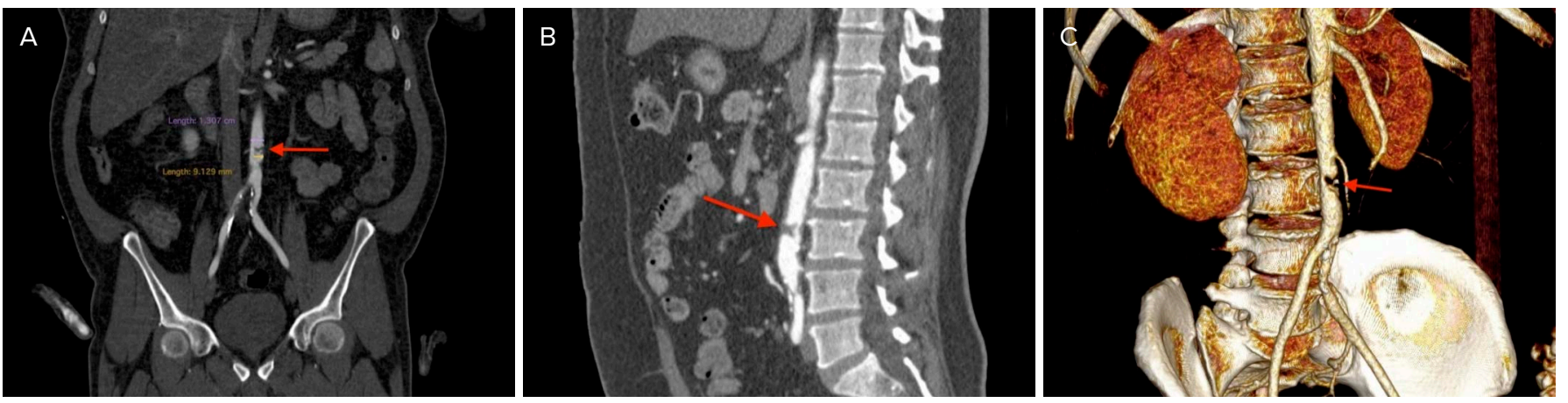

FIGURE 1. A - CT angiography coronal section, showing severe stenosis of the abdominal aorta; $\mathbf{B}$ - sagittal section showing the stenosis; C - 3D reconstruction showing severe isolated stenosis and subsequent repermeabilization without distal stenosis

\section{REFERENCES}

1. Schedel H, Wissgott C, Rademaker J, Steinkamp HJ. Primary Stent Placement for Infrarenal Aortic Stenosis: Immediate and Midterm Results. Journal of Vascular and Interventional Radiology. 2004;15:353-359.

2. Ocke Reis PE. Endovascular Treatment of Focal Infrarenal Aortic Stenosis with Absence of the Celiac Trunk - Case Report. J Vasc Endovasc Surg. 2016;:1:

3. Perrone-Filardi P, Costanzo P, Cesarano P, et al. Long abdominal aortic stenosis: A rare presentation of Takayasu arteritis treated with percutaneous stent implantation. The Journal of Thoracic and Cardiovascular Surgery. 2007;133:1647-1648.

4. Simons PCG, Nawijn AA, Bruijninckx CMA, Knippenberg B, de Vries $\mathrm{EH}$, van Overhagen $\mathrm{H}$. Long-term Results of Primary Stent Placement to Treat Infrarenal Aortic Stenosis. European Journal of Vascular and Endovascular Surgery. 2006;32:627-633. 